\title{
Vascular complications after radiosurgery for meningiomas
}

\author{
Kaveh Barami, M.D., Ph.D., ${ }^{1}$ Allison Grow, M.D., Ph.D., ${ }^{2}$ Steven Brem, M.D., ${ }^{3}$ \\ Elias Dagnew, M.D., ${ }^{1}$ AND ANDrew E. SloAn, M.D. ${ }^{3}$ \\ ${ }^{1}$ Memorial Neuroscience Center; ${ }^{2}$ Cyberknife Cancer Center, Memorial Hospital \\ Jacksonville; and ${ }^{3} \mathrm{H}$. Lee Moffitt Cancer Center \& Research Institute, Tampa, Florida
}

\begin{abstract}
$\checkmark$ During the past 25 years, radiosurgery has evolved as a primary treatment modality for certain meningiomas when resection would be associated with high patient morbidity. In addition, radiosurgery is now routinely used as an adjunctive therapy for residual or recurrent meningiomas after surgical removal. In this review the authors summarize the vascular complications that occur after radiosurgery for meningiomas as well as experimental study data that give insight into the pathogenesis of this complication. These data may be useful when discussing with patients the risk/benefit ratio of choosing among conservative management, radiosurgery, and surgery.
\end{abstract}

KeY WoRds • vascular complication • hemorrhage • occlusion • meningioma • radiosurgery

$\mathrm{M}$ OST MENINGIOMAS are histopathologically benign lesions, and yet their rate of recurrence increases if complete resection is not achieved..$^{21,31}$ Despite advances in the surgical approaches and techniques for removing these lesions, complete removal of parasellar, cavernous, orbital, and petroclival meningiomas and those near the major venous sinuses remains difficult and is associated with high morbidity. ${ }^{6,25}$ In reporting on the outcome of the aggressive removal of cavernous sinus meningiomas in 41 patients, DeMonte and colleagues ${ }^{6}$ noted permanent worsening of cranial nerve deficits in $15 \%$, new cranial deficits in $17 \%$, and cerebral ischemia in $7 \%$ of patients and a mortality rate of $7 \%$. As a result, stereotactic radiosurgery has emerged as the primary or adjuvant treatment modality in certain cases. In most reported series authors have used GKS as a therapeutic procedure. ${ }^{5,15,17,33}$ It is important to note that as the Gamma Knife prescription dose is delivered to the $50 \%$ isodose line, the intratumoral maximum dose reaches twice the prescription dose. Four-year actuarial tumor control of up to $92 \%$ has been reported. ${ }^{17}$

The rate of complications has ranged from 3 to $40 \%$ in different series. ${ }^{17,18}$ Most complications occur within 2 years of treatment and commonly include cranial nerve palsies in petroclival and cavernous sinus meningiomas,

Abbreviations used in this paper: $\mathrm{ACA}=$ anterior cerebral artery; $\mathrm{AVM}=$ arteriovenous malformation; $\mathrm{CT}=$ computed tomography; $\mathrm{GKS}=$ Gamma Knife surgery; ICA = internal carotid artery; MR = magnetic resonance. hemiparesis in convexity lesions, and hemianopia in occipital lobe meningiomas. ${ }^{5,8,15,33}$ Vascular complications following radiosurgery seem to be rare and can be classified as occlusion of vessels or hemorrhage. In this review article we summarize the reported cerebrovascular complications following radiosurgery for meningiomas as well as the experimental study data related to the histopathological findings of the cerebral vasculature after applying radiation.

\section{Vascular Complications After RAdiosurgery FOR MENingiomaS}

The vascular type of complications are rare among those reported after radiosurgery for meningiomas and can be categorized as hemorrhage or occlusion of vessels leading to ischemia. In 2000 Roche and colleagues ${ }^{28}$ reported the first vascular complication from radiosurgery for meningioma. In their study 92 patients with cavernous sinus meningiomas underwent GKS. One patient, a 32-year-old woman, presented with a transient contralateral central facial palsy 14 months after the radiosurgery treatment date. The prescription dose to the tumor margin was 18 Gy to the $50 \%$ isodose line. The estimated dose delivered to the intracavernous carotid artery was $36 \mathrm{~Gy}$. Doppler ultrasonography and MR imaging results showed occlusion of the intracavernous ICA.

Stafford and associates ${ }^{32}$ reported on their results in 190 patients with 206 meningiomas that had been treated using GKS; 77\% of the lesions involved the cranial base. 
Internal carotid artery stenosis occurred in two patients $(1 \%)$ with cavernous sinus meningiomas. One patient presented 60 months after the radiosurgical treatment date with ischemic symptoms contralateral to the meningioma. A 50\% stenosis of the cavernous segment of the ICA was seen. Another patient experienced cerebral infarction 35 months after radiosurgery, and total occlusion of the cavernous ICA was discovered. The calculated radiation dose to the affected arteries exceeded $25 \mathrm{~Gy}$. Both patients suffered permanent cerebroischemic deficits.

In reporting on their experience with GKS for cavernous sinus meningiomas (49 lesions), Pollock and Stafford ${ }^{26}$ described one patient (2\%) with an ischemic stroke causing hemiparesis and aphasia, which had occurred 39 months after treatment. The cavernous segment of the ICA was occluded on MR angiography at the time of the stroke. The exact dose delivered to the affected artery is unknown; however, the mean dose to the tumor margin was $15.9 \mathrm{~Gy}$, and the mean maximum radiation dose was $32.4 \mathrm{~Gy}$.

Kwon and associates ${ }^{16}$ reported on the incidence of intratumoral bleeding after GKS among a series of 173 meningiomas. Four patients suffered intratumoral hemorrhage. Two patients with tentorial meningiomas experienced intracystic hemorrhage occurring 1 and 5 years after treatment. Both received 20 Gy radiation as the prescription dose, with an intratumoral maximum of up to $40 \mathrm{~Gy}$. In another case, a patient with a temporal meningioma treated with $18 \mathrm{~Gy}$, intratumoral bleeding was found 2 years after treatment. Last, a patient with a cavernous petroclival lesion presented with progressive third and sixth cranial nerve palsies and was found to have intratumoral bleeding 8 years after GKS. The overall incidence of intratumoral bleeding was $2.3 \%$. On histological examination in three cases, no specific findings correlated with postradiosurgical changes; therefore, radiosurgery itself could not be shown to be a significant factor in the development of intratumoral bleeding.

Sanno and associates ${ }^{29}$ reported on the case of a patient with a frontoparietal parasagittal meningioma that had undergone a sarcomatous change. The patient presented with hemiparesis and aphasia 4 years after GKS. Computed tomography results showed intratumoral and peritumoral hemorrhage. The prescription dose was 30 Gy radiation. Last, Kim and colleagues ${ }^{13}$ reported on a patient harboring a tentorial meningioma who had presented with hemiparesis and a visual defect 3 years after treatment with GKS (prescription dose 15 Gy). She was found to have peritumoral hemorrhage on CT scanning.

The literature on vascular complications following radiosurgery is summarized in Table 1 .

\section{Experimental Study Data}

\section{Vasculopathy After Fractionated Radiotherapy}

The effect of radiation on cerebral vasculature has been reviewed. ${ }^{23}$ Authors of numerous reports have implicated radiation in the development of cerebrovascular injury including arterial stenosis/occlusion, ${ }^{3}$ aneurysm formation, ${ }^{2}$ necrosis, ${ }^{9}$ moyamoya disease,,${ }^{1}$ atherosclerosis, ${ }^{35}$ hemodynamic changes, ${ }^{20}$ and stroke. ${ }^{4}$ Experience with fractionated radiotherapy has shown that after administering radiation, the cerebral vasculature structure and function undergo distinct acute, intermediate, and late changes. ${ }^{27}$ Capillaries seem to be the most sensitive component of the vasculature and typically undergo pinocytosis and hypertrophic changes that correlate with endothelial proliferation and luminal narrowing. ${ }^{7}$ The component most radioresponsive to early injury is the endothelial cell. Ultrastructural study data have shown that, after a single dose of 20 Gy radiation, early changes occur in the capillary extracellular basement membrane and rough endoplasmic reticulum associated with cellular swelling. Platelet-fibrin thrombi develop a few days later. These pathological changes lead to increased permeability, which is responsible for severe cerebral edema after applying radiotherapy to the brain.

When aortic endothelial cells are exposed to ionizing radiation in vitro, within hours there is a change in endothelial cell F-actin distribution, cell retraction, and a dose-dependent increase in transendothelial flux of lowmolecular-weight solutes and albumin. ${ }^{23}$ These changes are accompanied by increased secretion of growth factors and chemoattractants as well as alterations in eicosanoid synthesis. After irradiation, surviving endothelial cells undergo cytoplasmic hypertrophy and giant cell formation with increased cellular adhesiveness for neutrophils. Last, the endothelial cells promote intercellular platelet deposition. Taken together, these changes ultimately lead to luminal narrowing and vessel occlusion.

TABLE 1

Literature review of studies on vascular complications after $G K S^{*}$

\begin{tabular}{|c|c|c|c|c|c|}
\hline Authors \& Year & Vascular Complication & $\begin{array}{l}\text { Time Delay } \\
(\mathrm{mos})\end{array}$ & $\begin{array}{l}\text { Radiation } \\
\text { Dose (Gy) }\end{array}$ & Tumor Location & Clinical Findings \\
\hline Roche et al., 2000 & ICA occlusion & 14 & $36 \dagger$ & cavernous sinus & temporary central facial palsy \\
\hline \multirow[t]{2}{*}{ Stafford et al., 2001} & $50 \%$ ICA stenosis & 60 & $>25$ & cavernous sinus & permanent cerebroischemic deficit \\
\hline & complete ICA occlusion & 35 & $>25$ & cavernous sinus & permanent cerebroischemic deficit \\
\hline \multirow[t]{4}{*}{ Kwon, 2002} & intracystic hemorrhage & 12 & 20 & tentorium & - \\
\hline & intracystic hemorrhage & 60 & 20 & tentorium & - \\
\hline & intratumoral bleeding & 24 & 18 & temporal & - \\
\hline & intratumoral bleeding & 96 & 17 & cavernous petroclival & CN III \& VI progressive palsy \\
\hline Kim et al., 2004 & peritumoral hemorrhage & 36 & 15 & tentorium & hemiparesis, visual defect \\
\hline Sanno et al., 2004 & intratumoral bleeding & 48 & 30 & frontoparietal & hemiparesis, aphasia \\
\hline Pollock et al., 2005 & ICA occlusion & 39 & $12-20$ & cavernous sinus & hemiparesis, aphasia \\
\hline
\end{tabular}


Authors of various experimental studies who have focused on the effects of radiation on cerebral vasculature have provided insight into the pathogenesis of vascular changes following radiotherapy. Arteries of the circle of Willis are examples of large muscular arteries. These vessels are less frequently injured than small or mediumsized arteries; however, the pathological consequences of the postradiation lesions may result in vessel rupture, stenosis, or occlusion. ${ }^{7}$ Rupture of these vessels can occur early due to wall necrosis. Delayed lesions are frequently fibrous intimal plaques that can narrow or occlude the lumen. This intimal fibrosis is either concentric or eccentric, causing significant reduction in the vessel diameter. The intimal cell may show slight atypia. The next most frequent type of lesion is a transmural healed necrosis. In this scenario, the vessel wall is replaced by fibrosis, and organized thrombi are frequent. The least common lesion to the arteries is similar to intimal fibrosis except that foam cells form part or all of the intimal plaque. Radiation vasculopathy seems to be dose and time dependent. ${ }^{34}$

\section{Vasculopathy After Radiosurgery}

Similar to the effects after fractionated radiotherapy, high doses of gamma radiation (100-300 Gy) to the cat basilar artery cause endothelial degeneration, desquamation with medial hyalinization, and necrosis. ${ }^{22}$ Omary and colleagues $^{24}$ irradiated the frontoparietal cortex in rats by using the Gamma Knife at a single dose of 120 Gy. Four weeks later, MR spectroscopy and gadopentetate dimeg- lumine-enhanced $\mathrm{T}_{1}$-weighted $\mathrm{MR}$ imaging were performed. Results of histological analysis showed extensive proliferation of capillaries associated with a moderate degree of astrocytosis. Gadopentetate dimeglumineenhanced $\mathrm{T}_{1}$-weighted MR imaging showed statistically significant signal intensity changes suggesting bloodbrain barrier disruption; however, no reproducible metabolic derangements of ischemia or necrosis were detected by MR spectroscopy. These results suggest that bloodbrain barrier disruption occurs in the early delayed phase of radiation injury; yet ischemia and necrosis have not occurred at this latency period.

Kamiryo and colleagues ${ }^{11}$ observed that the ACA was occluded after a single radiation dose of $100 \mathrm{~Gy}$ to the rat brain 20 months after GKS, causing cerebral infarction (Fig. 1). They observed arterial wall thickening with fibrosis, splitting of the internal elastic membrane, luminal organized thrombus, and migration of smooth-muscle cells into the thrombus. Endothelial injury seemed to be the initiating factor. In small arteries various changes occurred, including fibrosis and thrombosis, thickening of the smooth-muscle layer, lymphocytic infiltration, thickening of the vessel wall with fibrinous thrombosis, and leakage of fibrin into the surrounding tissue. Size-, dose-, and time-dependent effects on the cerebral vasculature were observed after administering radiation (25-100 Gy). Capillaries showed early hyalinization, arterioles revealed muscular thickening, small arteries had thrombus formation, and muscular arteries showed arterial wall fibrosis as

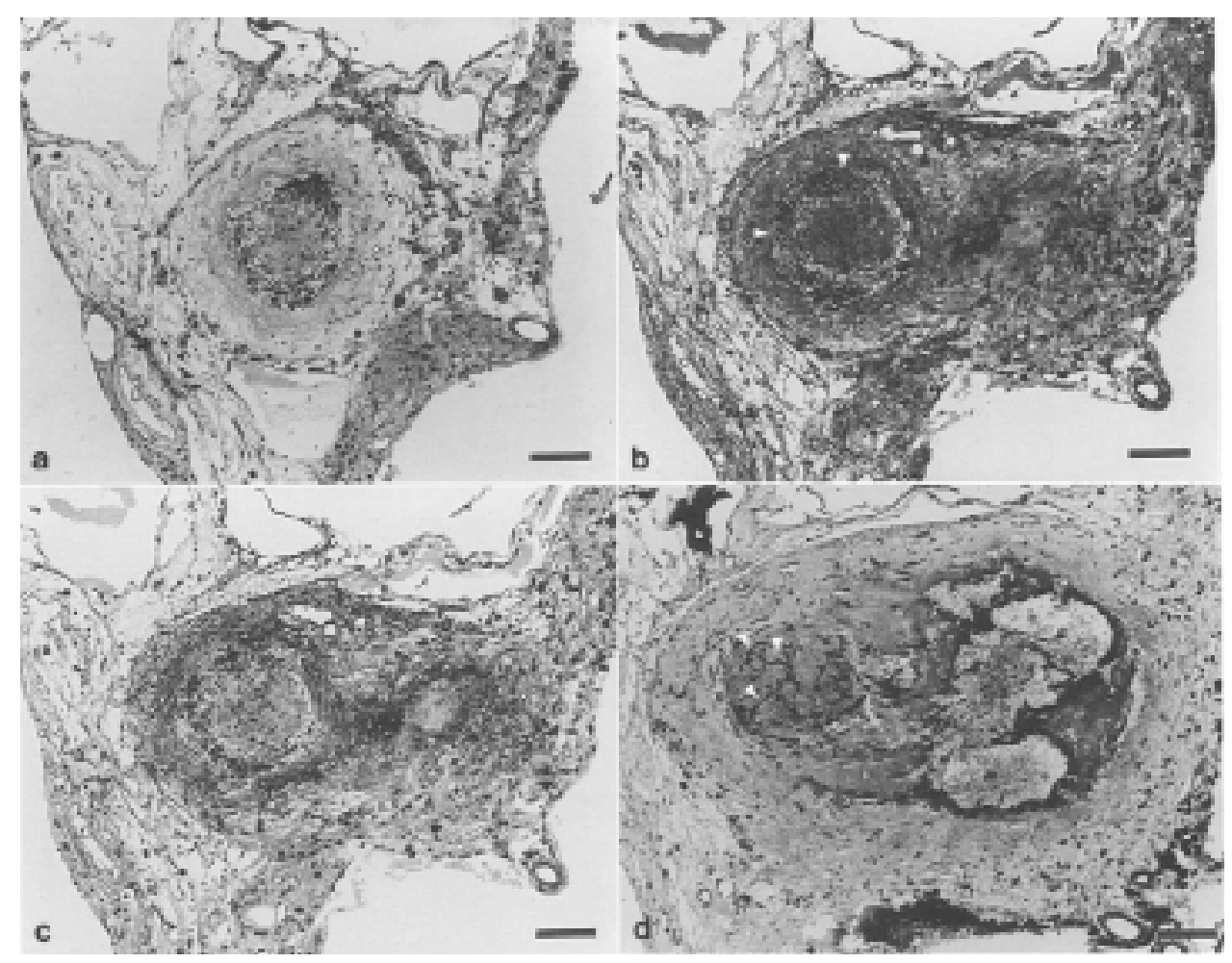

FIG. 1. Photomicrographs depicting the pathological features of the ACA 20 months after radiosurgery. a: Occlusion of the ACA with fibrosis of the wall and intraluminal thrombus. b: Disrupted internal elastic lamina (arrowheads). c: Thickening of the arterial wall with collagen fibers. d: Smooth-muscle cells (arrowheads) in the thrombus and fibrosis in the arterial wall. Bar $=100 \mu \mathrm{m}$. (Reprinted with permission from Kamiryo et al: Acta Neurochir (Wien) 138: 983-991, 1996.) 
well as thrombosis. The authors concluded that thrombosis caused by vascular damage is one of the mechanisms of artery occlusion and that gamma radiation causes damage to endothelial cells directly or indirectly.

In another study, Kamiryo and colleagues ${ }^{10}$ irradiated the parietal cortex of adult rats with GKS at different dosages. At 4 months postirradiation (75 Gy), they observed thickening of arteriolar walls by subintimal accumulation of fibrin and hyaline substance causing narrowing or occlusion of the lumen of capillaries and arterioles. Last, in another study involving Gamma Knife irradiation of the rat parietal cortex, Kamiryo and associates ${ }^{12}$ observed that delayed necrosis induced by gamma radiation is preceded by distinct microvascular changes such as capillary basement membrane thickening, suggesting a vascular mechanism for radiation necrosis.

Stereotactic radiosurgery has thus been shown to be an effective treatment for cerebral AVMs. The histopathological effects of radiosurgery are most prominent in abnormal vessels within the AVM and include early endothelial damage, intimal thickening, cellular degeneration, and vessel obliteration with hyalinization. ${ }^{30}$ Most likely, the radiation response mechanisms mediating vessel wall changes in AVMs are similar to those involved in normal arteries.

The literature regarding the pathophysiological processes of radiosurgery is sparse. To date, there have been no experimental studies elucidating the mechanism underlying intratumoral bleeding associated with radiosurgery. Spontaneous bleeding occurs in 1.3 to $2.4 \%$ of meningiomas. ${ }^{19} \mathrm{Kim}$ and colleagues ${ }^{14}$ have theorized that thrombosis, edema, vessel erosion, and rapid tumor growth lead to tumor infarction. As tumor infarction progresses, the intratumoral pressure increases and rupture of the peritumoral vessel eventually occurs. Further studies are needed to demonstrate these results. Experimental studies regarding the vascular changes following radiosurgery are summarized in Table 2.

\section{Conclusions}

Vascular complications following radiosurgery, including vessel occlusion and hemorrhage, are rare events, occurring 1.1 to $2.3 \%$ of the time. The incidence of vascu-

TABLE 2

Literature review of experimental studies on vascular reactions after $G K S^{*}$

\begin{tabular}{lcl}
\hline \hline \multicolumn{1}{c}{ Authors \& Year } & $\begin{array}{c}\text { Radiation } \\
\text { Dose (Gy) }\end{array}$ & \multicolumn{1}{c}{ Findings } \\
\hline $\begin{array}{l}\text { Nilsson et al., 1978† } \\
\text { Omary et al., 1995 }\end{array}$ & $100-300$ & $\begin{array}{l}\text { endothelial degeneration } \\
\text { BBB disruption, no ischemic } \\
\text { necrosis at 4 wks }\end{array}$ \\
$\begin{array}{l}\text { Kamiryo et al., 1996\$ } \\
\text { Kamiryo et al., 1996§ } \\
\text { Kamiryo et al., 2001 }\end{array}$ & $\begin{array}{c}50-120 \\
\text { occlusion of ACA at 20 mos } \\
\text { time and dose-dependent changes } \\
\text { capillary basement membrane } \\
\text { thickening }\end{array}$ \\
\hline
\end{tabular}

* Abbreviation: $\mathrm{BBB}=$ blood-brain barrier.

$\uparrow$ Study performed in the cat; all other studies performed in the rat.

\$ Reference 11 .

$\S$ Reference 10. lar occlusion after radiosurgery is 1 to $2 \%$ and occurs in a delayed fashion, usually 14 to 60 months after treatment. Lessons learned from fractionated radiotherapy and experimental studies with GKS suggest that occlusion of cerebral arteries after radiosurgery develops as a result of luminal narrowing associated with endothelial damage, which is most likely initiated by DNA damage. This phenomenon seems to be dose dependent. ${ }^{7,12}$ Radiation doses exceeding 25 Gy to the artery have been associated with occlusion in humans.

Although there are no established dosing guidelines for meningioma radiosurgery, commonly, an 18-Gy singlefraction dose equivalent is administered to the periphery, assuming coverage by the 65 to $80 \%$ isodose line. ${ }^{26,28,32}$ The incidence of hemorrhage after radiosurgery is $2.3 \%$ and occurs 1 to 8 years after treatment. The association between radiation and tumor/cerebral hemorrhage remains controversial and is likely mostly related to rapid postradiation tumor shrinkage leading to mechanical strain on vascular structures, rather than to histological changes intrinsic to the vasculature.

Cyberknife radiosurgery is emerging as a new modality to treat lesions all over the body. There have been no reported cases of vascular occlusion to date. Theoretically, the maximum intratumoral dose would be lower compared with that used in GKS given that the prescription dose is generally applied to the 65 to $85 \%$ isodose line as opposed to the $50 \%$ isodose line. Therefore, the incidence of vascular complications might be even lower than that for GKS.

Fractionated radiosurgery is emerging as a new method to deliver high doses to the tumor while affording some protection to nearby critical structures, because late effects on these normal tissues are reduced with smaller fraction sizes. Further studies are needed to determine whether the procedure will also improve clinical outcomes with respect to damaging cerebral vasculature leading to occlusion and ischemia.

In this brief report we summarized the clinical and experimental studies that have been focused on vascular complications after radiosurgery. With the increasing role of radiosurgery in the treatment of meningiomas, our data underscore the fact that the differential diagnosis in a patient with new neurological findings that appear in a delayed fashion must include vascular complication, and thus a full vascular workup is indicated, including Doppler ultrasonography, CT, MR imaging, MR angiography, and possibly cerebral angiography. In general, patients undergoing radiosurgery for meningiomas should be alerted to the possibility of vascular occlusion, which usually occurs on a delayed basis. This issue can be studied in the future by interval monitoring with MR angiography or CT angiography as noninvasive diagnostic modalities to assess the patency of the involved vessel.

The calculated risk of vascular complications following radiosurgery for meningiomas is significant when compared with the risks after microsurgery and thus aids in the final decision-making process in the management of these lesions. Patients should be counseled regarding these risks, but should not necessarily be deterred from the option of radiosurgery. Meningiomas located near sensitive vascular structures, such as the ICA or the venous sinuses, are precisely those that are most difficult to resect safely. The 
radiosurgical risk of vascular complications may in fact compare quite favorably to the surgical risk.

\section{References}

1. al-Amro A, Schultz H: Moyamoya vasculopathy after cranial irradiation-a case report. Acta Oncol 34:261-263, 1995

2. Azzarelli B, Moore J, Gilmor R, Muller J, Edwards M, Mealey $\mathrm{J}$ : Multiple fusiform intracranial aneurysms following curative radiation therapy for suprasellar germinoma. Case report. J Neurosurg 61:1141-1145, 1984

3. Bernstein M, Lumley M, Davidson G, Laperriere N, Leung P: Intracranial arterial occlusion associated with high-activity iodine-125 brachytherapy for glioblastoma. J Neurooncol 17: 253-260, 1993

4. Bowen J, Paulsen CA: Stroke after pituitary irradiation. Stroke 23:908-911, 1992

5. Chang JH, Chang JW, Choi JY, Park YG, Chung SS: Complications after gamma knife radiosurgery for benign meningiomas. J Neurol Neurosurg Psychiatry 74:226-230, 2003

6. DeMonte F, Smith HK, al-Mefty O: Outcome of aggressive removal of cavernous sinus meningiomas. J Neurosurg 81: 245-251, 1994

7. Fajardo LF, Berthrong M: Vascular lesions following radiation. Pathol Annu 23:297-330, 1988

8. Hakim R, Alexander E III, Loeffler JS, Shrieve DC, Wen P, Fallon MP, et al: Results of linear accelerator-based radiosurgery for intracranial meningiomas. Neurosurgery 42: 446-454, 1998

9. Ishibashi Y, Okada H, Mineura K, Kodama N: [A case of radiation necrosis with vascular changes on main cerebral arteries.] No Shinkei Geka 10:337-341, 1982 (Jpn)

10. Kamiryo T, Kassell NF, Thai QA, Lopes MB, Lee KS, Steiner L: Histological changes in the normal rat brain after gamma irradiation. Acta Neurochir (Wien) 138:451-459, 1996

11. Kamiryo T, Lopes MB, Berr SS, Lee KS, Kassell NF, Steiner L: Occlusion of the anterior cerebral artery after Gamma Knife irradiation in a rat. Acta Neurochir (Wien) 138:983-991, 1996

12. Kamiryo T, Lopes MB, Kassell NF, Steiner L, Lee KS: Radiosurgery-induced microvascular alterations precede necrosis of the brain neuropil. Neurosurgery 49:409-414, 2001

13. Kim CH, Kim DG, Paek SH, Chung HT, Choi YL, Chi JG: Delayed bleeding after gamma knife surgery for meningioma. Acta Neurochir (Wien) 146:741-742, 2004

14. Kim DG, Park CK, Paek SH, Choe GY, Gwak HS, Yoo H, et al: Meningioma manifesting intracerebral haemorrhage: a possible mechanism of haemorrhage. Acta Neurochir (Wien) 142:165-168, 2000

15. Kondziolka D, Levy EI, Niranjan A, Flickinger JC, Lunsford LD: Long-term outcomes after meningioma radiosurgery: physician and patient perspectives: J Neurosurg 91:44-50, 1999

16. Kwon Y, Ahn JS, Jeon SR, Kim JH, Kim CJ, Lee JK, et al: Intratumoral bleeding in meningioma after gamma knife radiosurgery. J Neurosurg 97 (5 Suppl):657-662, 2002

17. Lunsford LD: Contemporary management of meningiomas: radiation therapy as an adjuvant and radiosurgery as an alternative to surgical removal? J Neurosurg 80:187-190, 1994

18. Malik I, Rowe JG, Walton L, Radatz MW, Kemeny AA: The use of stereotactic radiosurgery in the management of meningiomas. Br J Neurosurg 19:13-20, 2005

19. Martinez-Lage JF, Poza M, Martinez M, Esteban JA, Antunez
MC, Sola J: Meningiomas with haemorrhagic onset. Acta Neurochir (Wien) 110:129-132, 1991

20. Mineura K, Sasajima T, Kowada M, Saitoh H, Shishido F. Case report: radiation-induced vasculopathy implicated by depressed blood flow and metabolism in a pineal glioma. Br J Radiol 66:727-733, 1993

21. Mirimanoff RO, Dosoretz DE, Linggood RM, Ojemann RG, Martuza RL: Meningioma: analysis of recurrence and progression following neurosurgical resection. J Neurosurg 62:18-24, 1985

22. Nilsson A, Wennerstrand J, Leksell D, Backlund EO: Stereotactic gamma irradiation of basilar artery in cat. Preliminary experiences. Acta Radiol Oncol Radiat Phys Biol 17: 150-160, 1978

23. O'Connor MM, Mayberg MR: Effects of radiation on cerebral vasculature: a review. Neurosurgery 46:138-151, 2000

24. Omary RA, Berr SS, Kamiryo T, Lanzino G, Kassell NF, Lee KS, et al: 1995 AUR Memorial Award. Gamma knife irradiation-induced changes in the normal rat brain studied with $1 \mathrm{H}$ magnetic resonance spectroscopy and imaging. Acad Radiol 2:1043-1051, 1995

25. Origitano TC, al-Mefty O, Leonetti JP, DeMonte F, Reichman $\mathrm{OH}$ : Vascular considerations and complications in cranial base surgery. Neurosurgery 35:351-363, 1994

26. Pollock BE, Stafford SL: Results of stereotactic radiosurgery for patients with imaging defined cavernous sinus meningiomas. Int J Radiat Oncol Biol Phys 62:1427-1431, 2005

27. Reinhold HS, Keyeux A, Dunjic A, Jovanovic D, Maisin JR: The influence of radiation on blood vessels and circulation. XII. Discussion and conclusions. Curr Top Radiat Res Q 10: 185-198, 1974

28. Roche P, Regis, J, Dufour H, et al: Gamma knife radiosurgery in the management of cavermous sinus meningiomas. J Neurosurg (suppl 3) 93:68-73, 2000

29. Sanno N, Hayashi S, Shimura T, Maeda S, Teramoto A: Intracranial osteosarcoma after radiosurgery-case report. Neurol Med Chir (Tokyo) 44:29-32, 2004

30. Schneider BF, Eberhard DA, Steiner LE: Histopathology of arteriovenous malformations after gamma knife radiosurgery. J Neurosurg 87:352-357, 1997

31. Simpson D: The recurrence of intracranial meningiomas after surgical treatment. J Neurol Neurosurg Psychiatry 20:22-39, 1957

32. Stafford SL, Pollock BE, Foote RL, Link MJ, Gorman DA, Schomberg PJ, et al: Meningioma radiosurgery: tumor control, outcomes, and complications among 190 consecutive patients. Neurosurgery 49:1029-1037, 2001

33. Subach BR, Lunsford LD, Kondziolka D, Maitz AH, Flickinger JC: Management of petroclival meningiomas by stereotactic radiosurgery. Neurosurgery 42:437-445, 1998

34. Valk PE, Dillon WP: Radiation injury of the brain. AJNR Am J Neuroradiol 12:45-62, 1991

35.Werner MH, Burger PC, Heinz ER, Friedman AH, Halperin EC, Schold SC Jr: Intracranial atherosclerosis following radiotherapy. Neurology 38:1158-1160, 1988

Manuscript submitted September 15, 2006.

Accepted January 18, 2007.

Address reprint requests to: Kaveh Barami, M.D., Ph.D., 2835 Casa Del Rio Terrace, Jacksonville, Florida 32257. email: kbarami@yahoo.com. 